\title{
The new intermediate long-bursting source XTE J1701-407
}

\author{
M. Falanga ${ }^{1,2}$, A. Cumming ${ }^{3}$, E. Bozzo ${ }^{4,5}$, and J. Chenevez ${ }^{6}$ \\ ${ }^{1}$ CEA Saclay, DSM/IRFU/Service d'Astrophysique (CNRS FRE 2591), 91191 Gif-sur-Yvette, France \\ e-mail: mfalanga@cea.fr \\ 2 AIM - Unité Mixte de Recherche CEA - CNRS - Université Paris 7, Paris, France \\ 3 Physics Department, McGill University, 3600 rue University, Montreal QC, H3A 2T8, Canada \\ 4 INAF - Osservatorio Astronomico di Roma, Via Frascati 33, 00044 Rome, Italy \\ 5 Dipartimento di Fisica - Universitá di Roma Tor Vergata, via della Ricerca Scientifica 1, 00133 Rome, Italy \\ ${ }^{6}$ National Space Institute, Technical University of Denmark, Juliane Maries Vej 30, 2100 Copenhagen, Denmark \\ Received 6 October 2008 / Accepted 17 December 2008
}

\section{ABSTRACT}

\begin{abstract}
Aims. XTE J1701-407 is a newly discovered X-ray transient source. We investigate its flux variability and study the intermediate long- and short-bursts discovered by Swift on July 17, and 27, 2008, respectively.

Methods. Only one intermediate long-burst, of duration $\approx 18 \mathrm{~min}$ and ten days later a short burst, have been recorded from XTE J1701407. We analyzed the public available data from Swift and RXTE, and compared the observed properties of the intermediate long-burst with theoretical ignition condition and light curves to investigate the possible nuclear-burning processes.

Results. The intermediate long-burst may have exhibited a photospheric radius expansion, allowing us to derive the source distance at $6.2 \mathrm{kpc}$, assuming the empirically derived Eddington luminosity for pure helium. The intermediate long-burst decay was described most accurately by using two exponential functions with e-folding times of $\tau_{1}=40 \pm 3 \mathrm{~s}$ and $\tau_{2}=221 \pm 9 \mathrm{~s}$. The bursts occurred at a persistent luminosity of $L_{\mathrm{per}}=8.3 \times 10^{36} \mathrm{erg} \mathrm{s}^{-1}(\approx 2.2 \%$ of the Eddington luminosity). For the intermediate long-burst, the mass accretion rate per unit area onto the neutron star was $\dot{m} \approx 4 \times 10^{3} \mathrm{~g} \mathrm{~cm}^{-2} \mathrm{~s}^{-1}$, and the total energy released was $E_{\text {burst }} \approx 3.5 \times 10^{40} \mathrm{erg}$. This corresponds to an ignition column depth of $y_{\mathrm{ign}} \approx 1.8 \times 10^{9} \mathrm{~g} \mathrm{~cm}^{-2}$, for a pure helium burning. We find that the energetics of this burst can be modeled in different ways, as (i) pure helium ignition, as the result of either pure helium accretion or depletion of hydrogen by steady burning during accumulation; or (ii) as ignition of a thick layer of hydrogen-rich material in a source of low metallicity. However, comparison of the burst duration with model light curves suggests that hydrogen burning plays an important role during the burst, and therefore that this source is a low accretion-rate burster with a low metallicity in the accreted material.
\end{abstract}

Key words. stars: binaries: close - stars: individual: XTE J1701-407 - stars: neutron - X-rays: bursts

\section{Introduction}

Type-I X-ray bursts are among the most evident signatures of the presence of a neutron star (NS) in low mass X-ray binaries. These bursts are thermonuclear explosions that occur on the surface of accreting NSs and are triggered by unstable hydrogen and/or helium burning (see, e.g., Lewin et al. 1993; Strohmayer \& Bildsten 2006, for reviews). Type-I X-ray bursts were predicted theoretically by Hansen \& Van Horn (1975), and several thousand bursts have been observed to date (see, e.g., Cornelisse et al. 2003; Galloway et al. 2006a; Chelovekov et al. 2006). From the duration of the bursts measured by their decay parameter $\tau$ (see e.g., Galloway et al. 2006a) three main branches are distinguishable: normal bursts, intermediate long-bursts, and superbursts (see Fig. 7 in Falanga et al. 2008, and references therein). These bursts can be described in terms of different fuel types, accretion rates, and they, therefore, also exhibit different recurrence times (e.g., Strohmayer \& Bildsten 2006; Cumming \& Macbeth 2004; Galloway et al. 2006a). Thanks to the long Galactic plane scan carried out with BeppoSAX, INTEGRAL, and Swift, a number of rare, intermediate long-bursts and superbursts have been observed. In most cases, the rise of the burst was $\approx 1 \mathrm{~s}$, whereas the decay was approximately exponential, with a duration of a few seconds for normal bursts, tens of minutes for intermediate long-bursts, and up to several hours for superbursts (e.g., Kuulkers 2004; in 't Zand et al. 2004; Molkov et al. 2005; in 't Zand et al. 2005; Chenevez et al. 2006, 2007;
Falanga et al. 2008). The recurrence time of type-I X-ray busts ranges from few hours to years, depending on the nuclear reactions involved (see, e.g., Lewin et al. 1993; Strohmayer \& Bildsten 2006, for reviews).

In this paper, we report on an intermediate long-burst from the X-ray transient XTE J1701-407. This source was discovered by the Rossi X-ray Timing Explorer (RXTE) during a routine Galactic bulge scan on June 8, 2008 (Markwardt et al. 2008a). At the time of discovery, the source spectrum was most accurately reproduced by an absorbed power-law model with photon index $\approx 2.2$ and a neutral absorption column of $\approx 3.4 \times 10^{22} \mathrm{~cm}^{-2}$, the measured flux was $\approx 1.4 \times 10^{-10} \mathrm{erg} \mathrm{cm}^{-2} \mathrm{~s}^{-1}$ in the $2-10 \mathrm{keV}$ band (Markwardt et al. 2008a). Swift/XTE follow-up observations on June 11, 2008 found both spectral and source flux measurements consistent with the $R X T E / P C A$ results (Degenaar \& Wijnands 2008). On July 17, 2008, the Swift/BAT camera detected a short flare consistent with the position of XTE J1701407, and 297 s later Swift/XRT measured a decaying X-ray flux (Barthelmy et al. 2008). Based on this BAT data, the Xray spectrum was consistent with those observed for thermonuclear X-ray bursts, therefore, this flare was proposed to be a type-I burst (Markwardt et al. 2008b). Based on the Swift/XRT time-resolved spectrum during flux decay, Linares et al. (2008a) measured the cooling tail and confirmed that the flare was an intermediate, long, type-I X-ray burst. This led to the classification of XTE J1701-407 as a NS low-mass X-ray binary (Linares et al. 2008a,b). Ten days later, i.e., on July 27, BAT observed 
Table 1. Log of RXTE and Swift observations and best-fit spectral parameters of the persistent emission. An absorbed power-law model is used to fit these spectra.

\begin{tabular}{|c|c|c|c|c|c|c|c|c|}
\hline Instrument & $\begin{array}{l}\text { Date } \\
\text { MJD }\end{array}$ & $\begin{array}{c}\text { Exp. } \\
\text { ks }\end{array}$ & $\begin{array}{c}N_{\mathrm{H}} \\
10^{22} \mathrm{~cm}^{-2}\end{array}$ & $\Gamma$ & $\begin{array}{l}\text { Flux }_{2-10 \mathrm{keV}}^{a} \\
\operatorname{erg~cm}^{-2} \mathrm{~s}^{-1}\end{array}$ & $\begin{array}{l}\text { Flux }_{2-20 \mathrm{keV}}^{a} \\
\operatorname{erg~cm}^{-2} \mathrm{~s}^{-1}\end{array}$ & $\begin{array}{l}\text { Flux }_{0.3-100 \mathrm{keV}}^{a} \\
\operatorname{erg~cm}^{-2} \mathrm{~s}^{-1}\end{array}$ & $\chi_{\text {red }}^{2}$ \\
\hline Swift $t^{b}$ & 54628.66385 & 0.4 & $4.0_{-0.6}^{+0.7}$ & $2.2_{-0.3}^{+0.3}$ & $2.8 \pm 0.3 \mathrm{e}-10$ & $3.7 \pm 0.5 e-10$ & $10.2 \pm 2 \mathrm{e}-10$ & 0.8 \\
\hline$R X T E$ & 54633.24637 & 2.0 & $3.4_{-2}^{+1.0}$ & $2.4_{-0.2}^{+0.3}$ & $4.5 \pm 0.4 \mathrm{e}-10$ & $5.6 \pm 0.4 \mathrm{e}-10$ & $17.5 \pm 7 e-10$ & 1.2 \\
\hline$R X T E$ & 54634.42452 & 2.5 & $3.4_{-1.2}^{+1.2}$ & $2.4_{-0.2}^{+0.1}$ & $3.4 \pm 0.2 \mathrm{e}-10$ & $4.3 \pm 0.2 \mathrm{e}-10$ & $12.8 \pm 2 \mathrm{e}-10$ & 0.7 \\
\hline$R X T E$ & 54635.27545 & 2.0 & $3.3_{-2.2}^{+2.1}$ & $2.2_{-0.2}^{+0.1}$ & $2.7 \pm 0.2 \mathrm{e}-10$ & $3.6 \pm 0.2 \mathrm{e}-10$ & $9.4 \pm 1.6 \mathrm{e}-10$ & 0.8 \\
\hline$R X T E$ & 54638.80878 & 3.5 & $3.4_{-0.9}^{+0.8}$ & $2.2_{-0.1}^{-0.2}$ & $1.9 \pm 0.1 \mathrm{e}-10$ & $2.6 \pm 0.1 \mathrm{e}-10$ & $6.8 \pm 1.1 \mathrm{e}-10$ & 0.9 \\
\hline$R X T E$ & 54639.85563 & 3.0 & $3.4_{-0.7}^{+0.9}$ & $2.3_{-0.1}^{+0.1}$ & $2.1 \pm 0.1 \mathrm{e}-10$ & $2.8 \pm 0.1 \mathrm{e}-10$ & $7.8 \pm 1.4 \mathrm{e}-10$ & 1.2 \\
\hline RXTE & 54640.90230 & 3.5 & $3.5_{-0.7}^{+0.7}$ & $2.2_{-0.1}^{+0.1}$ & $1.9 \pm 0.1 \mathrm{e}-10$ & $2.6 \pm 0.1 \mathrm{e}-10$ & $7.1 \pm 1.1 \mathrm{e}-10$ & 1.2 \\
\hline Swift $t^{b, c}$ & 54664.62684 & 0.6 & $3.1_{-0.4}^{-0.4}$ & $1.9_{-0.2}^{-0.1}$ & $4.9 \pm 0.3 e-10$ & $7.2 \pm 0.6 \mathrm{e}-10$ & $18.3 \pm 3 e-10$ & 1.1 \\
\hline RXTE & 54665.10546 & 3.0 & $3.7_{-2}^{+1.2}$ & $2.6_{-0.1}^{+0.2}$ & $4.9 \pm 0.6 \mathrm{e}-10$ & $5.9 \pm 0.7 e-10$ & $20.0 \pm 4 \mathrm{e}-10$ & 1.6 \\
\hline$R X T E$ & 54669.24527 & 1.8 & $2.9_{-2}^{+1.2}$ & $2.5_{-0.2}^{+0.1}$ & $4.8 \pm 0.8 \mathrm{e}-10$ & $5.8 \pm 0.9 \mathrm{e}-10$ & $18.0 \pm 3 e-10$ & 1.6 \\
\hline RXTE & 54670.99462 & 3.3 & $3.9_{-2}^{+1.2}$ & $2.5_{-0.2}^{+0.1}$ & $5.5 \pm 0.7 e-10$ & $6.5 \pm 0.6 \mathrm{e}-10$ & $23.2 \pm 4 \mathrm{e}-10$ & 1.5 \\
\hline RXTE & 54672.97638 & 1.7 & $4.1_{-2}^{+1.2}$ & $2.7_{-0.2}^{+0.2}$ & $4.9 \pm 0.5 e-10$ & $5.8 \pm 0.6 e-10$ & $21.5 \pm 5 e-10$ & 1.3 \\
\hline$R X T E$ & 54674.73749 & 1.8 & $4.2_{-2}^{+1.2}$ & $2.7_{-0.3}^{-0.2}$ & $4.6 \pm 0.7 \mathrm{e}-10$ & $5.5 \pm 0.6 \mathrm{e}-10$ & $20.8 \pm 5 e-10$ & 1.6 \\
\hline Swift ${ }^{b, d}$ & 54674.94054 & 0.1 & $3.4_{-0.4}^{+0.4}$ & $2.0_{-0.4}^{+0.4}$ & $7.3 \pm 0.5 e-10$ & $8.3 \pm 0.6 \mathrm{e}-10$ & $23.3 \pm 4 \mathrm{e}-10$ & 1.4 \\
\hline RXTE & 54677.47471 & 3.5 & $4.2_{-1.2}^{+1.4}$ & $2.6_{-0.2}^{+0.3}$ & $4.5 \pm 0.7 e-10$ & $5.3 \pm 0.8 \mathrm{e}-10$ & $18.3 \pm 3 e-10$ & 1.2 \\
\hline RXTE & 54678.59119 & 3.3 & $4.4_{-1}^{+2.2}$ & $2.3_{-0.1}^{+0.3}$ & $4.3 \pm 0.7 e-10$ & $5.0 \pm 0.8 \mathrm{e}-10$ & $17.5 \pm 4 \mathrm{e}-10$ & 1.5 \\
\hline RXTE & 54679.63915 & 3.2 & $4.1_{-2}^{+1.2}$ & $2.7_{-0.3}^{+0.1}$ & $4.0 \pm 0.5 e-10$ & $5.1 \pm 0.6 \mathrm{e}-10$ & $14.9 \pm 5 e-10$ & 1.3 \\
\hline$R X T E$ & 54680.29712 & 2.1 & $2.9_{-1.4}^{+1.2}$ & $2.5_{-0.3}^{+0.3}$ & $3.7 \pm 0.5 e-10$ & $4.3 \pm 0.6 \mathrm{e}-10$ & $13.2 \pm 3 e-10$ & 1.5 \\
\hline$R X T E$ & 54681.86101 & 3.5 & $3.6_{-2}^{+1.2}$ & $2.3_{-0.2}^{+0.3}$ & $3.3 \pm 0.5 e-10$ & $3.7 \pm 0.6 \mathrm{e}-10$ & $14.7 \pm 3 \mathrm{e}-10$ & 1.1 \\
\hline$R X T E$ & 54682.71212 & 7.0 & $2.3_{-0.9}^{+0.9}$ & $2.5_{-0.1}^{-0.2}$ & $1.5 \pm 0.04 \mathrm{e}-10$ & $1.9 \pm 0.05 e-10$ & $6.5 \pm 1.4 \mathrm{e}-10$ & 1.1 \\
\hline$R X T E$ & 54683.89045 & 3.5 & $2.2_{-1.2}^{+0.9}$ & $2.2_{-0.2}^{-0.1}$ & $0.95 \pm 0.07 e-10$ & $1.2 \pm 0.5 \mathrm{e}-10$ & $3.4 \pm 0.6 \mathrm{e}-10$ & 0.8 \\
\hline$R X T E$ & 54684.93786 & 1.8 & $2.2_{-1}^{-1.9}$ & $2.2_{-0.2}^{-0.2}$ & $0.89 \pm 0.03 e-10$ & $1.4 \pm 0.3 \mathrm{e}-10$ & $3.2 \pm 0.5 e-10$ & 0.9 \\
\hline$R X T E$ & 54685.80434 & 1.6 & $2.0_{-0.6}^{+1.0}$ & $2.2_{-0.1}^{-0.2}$ & $0.84 \pm 0.06 \mathrm{e}-10$ & $1.1 \pm 0.07 \mathrm{e}-10$ & $3.0 \pm 0.6 \mathrm{e}-10$ & 1.2 \\
\hline$R X T E$ & 54686.31211 & 2.5 & $1.3_{-0.4}^{-0.6}$ & $2.3_{-0.1}^{+0.1}$ & $0.94 \pm 0.06 \mathrm{e}-10$ & $1.2 \pm 0.6 \mathrm{e}-10$ & $3.5 \pm 0.8 \mathrm{e}-10$ & 0.8 \\
\hline$R X T E$ & 54687.04156 & 0.7 & $4.0_{-2}^{-0.4}$ & $2.5_{-0.2}^{-0.1}$ & $1.2 \pm 0.1 \mathrm{e}-10$ & $1.5 \pm 0.2 \mathrm{e}-10$ & $5.2 \pm 1.2 \mathrm{e}-10$ & 0.7 \\
\hline$R X T E$ & 54687.35989 & 2.7 & $2.7_{-1.2}^{+1.2}$ & $2.3_{-0.1}^{+0.2}$ & $1.1 \pm 0.06 \mathrm{e}-10$ & $1.5 \pm 0.06 \mathrm{e}-10$ & $4.4 \pm 0.9 \mathrm{e}-10$ & 1.3 \\
\hline$R X T E$ & 54687.43638 & 2.2 & $2.7_{-1.3}^{+1.3}$ & $2.3_{-0.1}^{+0.1}$ & $1.1 \pm 0.07 \mathrm{e}-10$ & $1.4 \pm 0.08 \mathrm{e}-10$ & $4.4 \pm 1.0 \mathrm{e}-10$ & 1.5 \\
\hline$R X T E$ & 54688.34471 & 2.4 & $2.7_{-1.2}^{+1.2}$ & $2.4_{-0.1}^{+0.1}$ & $1.2 \pm 0.05 \mathrm{e}-10$ & $1.6 \pm 0.05 e-10$ & $4.9 \pm 2 \mathrm{e}-10$ & 1.2 \\
\hline$R X T E$ & 54690.23786 & 2.2 & $2.3_{-1.0}^{+1.2}$ & $2.3_{-0.1}^{+0.1}$ & $1.4 \pm 0.06 \mathrm{e}-10$ & $1.9 \pm 0.1 \mathrm{e}-10$ & $5.5 \pm 1 \mathrm{e}-10$ & 1.4 \\
\hline$R X T E$ & 54691.67712 & 1.8 & $1.9_{-1.0}^{+1.0}$ & $2.3_{-0.1}^{+0.1}$ & $1.5 \pm 0.07 \mathrm{e}-10$ & $2.0 \pm 0.07 \mathrm{e}-10$ & $5.6 \pm 1.5 e-10$ & 1.6 \\
\hline$R X T E$ & 54692.13508 & 1.6 & $1.7_{-1}^{+1}$ & $2.2_{-0.1}^{+0.1}$ & $1.5 \pm 0.07 \mathrm{e}-10$ & $2.0 \pm 0.06 \mathrm{e}-10$ & $5.6 \pm 1.1 \mathrm{e}-10$ & 1.3 \\
\hline$R X T E$ & 54692.74785 & 1.2 & $3.1_{-1.3}^{+1.3}$ & $2.3_{-0.1}^{+0.1}$ & $1.8 \pm 0.07 \mathrm{e}-10$ & $2.3 \pm 0.1 \mathrm{e}-10$ & $6.8 \pm 1.2 \mathrm{e}-10$ & 1.3 \\
\hline$R X T E$ & 54696.71397 & 0.5 & $3.2_{-1.5}^{+2}$ & $2.3_{-0.1}^{+0.1}$ & $2.1 \pm 0.1 \mathrm{e}-10$ & $2.8 \pm 0.2 \mathrm{e}-10$ & $8.2 \pm 1.6 \mathrm{e}-10$ & 1.1 \\
\hline
\end{tabular}

${ }^{a}$ Unabsorbed flux; ${ }^{b}$ Swift/XRT was operating in WT mode; ${ }^{c, d}$ intermediate long and short pre-burst persistent emission, respectively.

in the $15-350 \mathrm{keV}$ band, a short burst lasting $\approx 10 \mathrm{~s}$ (Sakamoto et al. 2008). Kilohertz quasi-periodic oscillations were observed at $\approx 1150 \mathrm{~Hz}$ (see Strohmayer et al. 2008, for details). The most accurate position of the source was provided by Swift at $\alpha_{\mathrm{J} 2000}=17^{\mathrm{h}} 01^{\mathrm{m}} 44^{\mathrm{s}} .3$ and $\delta_{\mathrm{J} 2000}=-40^{\circ} 51^{\prime} 29^{\prime \prime} .9$ with an estimated accuracy of 1'.7 (Starling \& Evans 2008). No infrared counterpart candidate was found at this position (Kaplan \& Chakrabarty 2008).

\section{Data analysis and results}

In the present study, we used publicly available data from Swift and RXTE observatories. The dataset include RXTE target of opportunity observations, performed after the discovery of the source (observation ID. 93444), as well as Swift follow-up observations (ID. 0031221001, 0031221002, and 00317205001). The Swift data set also includes the intermediate long-burst discovered on July 17, (ID. 00317205000) and the short burst from July 27, 2008 (ID. 00318166000). The total effective exposure time is $75.4 \mathrm{ks}$ (30 pointings) and $1.1 \mathrm{ks}$ for $R X T E / \mathrm{PCA}$ and Swift, respectively. In Table 1, we report the detailed observations log. Data reduction of the RXTE Proportional Counter
Array (PCA; 2-60 keV, Jahoda et al. 1996) and the High Energy X-ray Timing Experiment (HEXTE; 15-250 keV, Rothschild et al. 1998) was performed according to the default selection criteria for background-subtraction, light-curve, and spectrum extraction. We used only PCU2 data. We used Swift/XRT data in both Windowed Timing (WT) and Photon Counting (PC) mode. However, in the following, we report only on WT data, since we found that PC data were strongly affected by pile-up, and quasi-simultaneous observations carried out in WT mode were available. We reduced all the XRT data by using the version of the xrtpipeline (version 0.11.6) included in the Heasoft package 6.4, and the latest calibration files available. Source events in WT mode were extracted from rectangle regions with widths of 40 pixels and heights of 20 pixels $^{1}$. Ancillary response files were generated with xrtmkarf and accounted for different extraction regions, vignetting, and point-spread function. For the Swift/BAT data analysis, we used the batgrbproduct tool included in the Heasoft package 6.4. Time-resolved spectra and light curves (see Sect. 2.2) were extracted by

\footnotetext{
1 See also the XRT analysis manual at http://swift.gsfc.nasa. gov/docs/swift/analysis/xrt_swguide_v1_2.pdf
} 


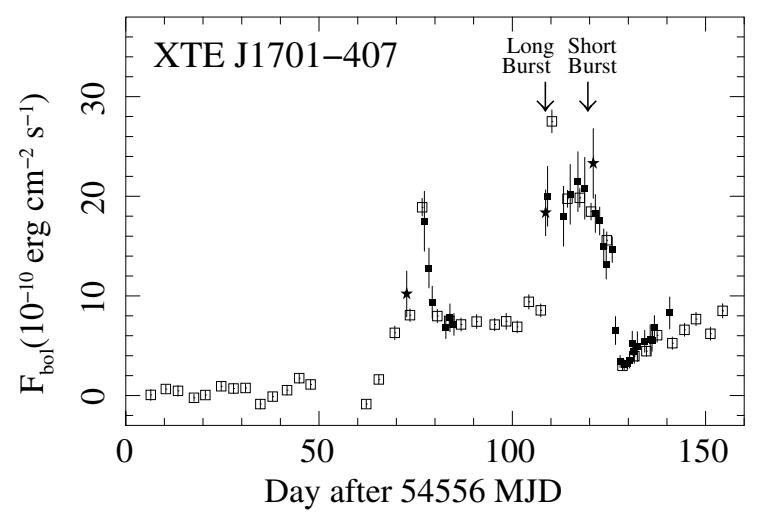

Fig. 1. Flux history of the X-ray transient XTE J1701-407. Open squares represent fluxes derived from $R X T E$ bulge observations in the period from March 31, to September 2, 2008. Fluxes derived from RXTE/PCA and Swift/XRT target of opportunity and follow-up observations are marked with filled squares and stars, respectively. The arrow indicates the time of the type-I X-ray burst.

using the batbinevt, batupdatephakw, batphasyserr, and batdrmgen tools.

\subsection{Persistent emission}

Spectra obtained from $R X T E / \mathrm{PCA}$ and Swift/XRT observations were fit separately by using a photoelectrically-absorbed powerlaw model. The best-fit model parameters for the absorption column, $N_{\mathrm{H}}$, are between $1.7 \pm 1$ and $4.4 \pm 1.2 \times 10^{22} \mathrm{~cm}^{-2}$ and a power-law index $\Gamma=2.0 \pm 0.1-2.7 \pm 0.3$ the corresponding unabsorbed fluxes are between $1.1 \pm 0.07 \times 10^{-10}$ and $8.3 \pm 0.6 \times 10^{-10}$ in the $2-20 \mathrm{keV}$ energy band. In Table 1 , we report all the persistent spectral parameters. All uncertainties correspond to a $90 \%$ confidence level. PCA spectra were extracted in the $2-20 \mathrm{keV}$ energy band; the source was detected only at low significance in the HEXTE (15-50 keV band). A fit to the joint broad-band PCA/HEXTE (2-50 keV) spectrum did not lead to a significant improvement in the determination of the model parameters; in the following we therefore, report only results for the PCA data. Swift/XRT spectra were extracted in the $2-10 \mathrm{keV}$ band. All the measured unabsorbed fluxes were extrapolated to the 0.3-100 keV band by generating dummy responses (XSPEC version 11.3.2ag), and are shown in Fig. 1. We also included in this figure fluxes derived from $R X T E$ bulge observations ${ }^{2}$ (Swank \& Markwardt 2001). In these cases, the conversion between $R X T E$ count rate and flux was obtained using the spectra results and the values reported by Markwardt et al. (2008a).

The ligh curve and spectral analysis illustate that XTE J1701-407 experienced flaring activity for a few days, during which the X-ray flux varied by over a factor of $2-3$. This variability can be ascribed to changes in the mass accretion rate or in the position of the source within its colour-colour diagram (CCD, see e.g., Hasinger \& van der Klis 1989). The latter possibility is investigated in Fig. 2. The CCD was produced by using background-subtracted $R X T E / P C A$ ligh curves with a $516 \mathrm{~s}$ time resolution. The soft colour is defined to be the logarithm of the ratio of the count rates in the energy band $2.1-3.7 \mathrm{keV}$ to that for band $3.7-5.7 \mathrm{keV}$, whereas for the hard colour the ratio of the energy band $5.7-9.8 \mathrm{keV}$ to $9.8-18.9 \mathrm{keV}$

\footnotetext{
2 http://lheawww.gsfc.nasa.gov/users/craigm/galscan/ main. html
}

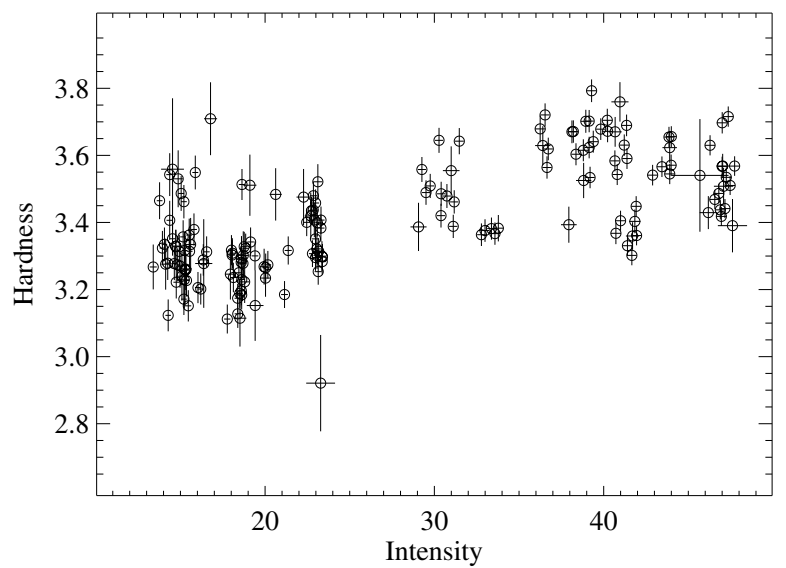

Fig. 2. HID of XTE J1701-407. The hardness is the ratio of the count rates in the $5.7-18.9 \mathrm{keV}$ to $2.1-5.7 \mathrm{keV}$ and the intensity is in the 2.1-18.9 $\mathrm{keV}$ count rate. Each point corresponds to $516 \mathrm{~s}$ of integration time.

is used. No obvious transition between a hard to a soft state is observed. To reduce the errors in the colours, we generated a hard intensity diagram (HID) based on the net count rates in the $2.1-5.7 \mathrm{keV}$ and $5.7-18.9 \mathrm{keV}$ energy ranges with a $516 \mathrm{~s}$ time bin. However, the source behaviour was not traced with significantly higher quality in the HID, because the statistical uncertainties are not reduced significantly along the two axes. In Fig. 2, we show the HID. Future observations are thus required to investigate further the origin of flux variations in XTE J1701-407.

\subsection{Intermediate long-burst light curves and spectra}

In Fig. 3, we show the Swift/BAT 15-25 keV (upper panel) and XRT $0.3-10 \mathrm{keV}$ (lower panel) light curve of the intermediate long-burst. The burst start time is the time at which the BAT $\mathrm{X}$-ray intensity of the source increased to $10 \%$ of its peak value above the persistent intensity level. The XRT light curve starts with a delay of $133 \mathrm{~s}$ after the beginning of the burst observed by BAT. The BAT light curve exhibit a slow rise time ${ }^{3}$ of $\approx 45 \mathrm{~s}$. The XRT decay time from the burst is best-fit by using two exponential functions with e-folding times of $\tau_{1}=44 \pm 8 \mathrm{~s}$ and $\tau_{2}=271 \pm 21 \mathrm{~s}$, respectively (see also Linares et al. 2008a). The total duration of the burst, i.e. the time to evolve away from and return to the persistent state, was of $\approx 86 \mathrm{~s}$ and $\approx 18 \mathrm{~min}$ in the BAT (15-25 keV) and the XRT (0.3-10 keV) light curves, respectively.

Type-I X-ray bursts are produced by unstable burning of accreted matter on the surface of the NS. The emission can be described well by black-body radiation with temperatures, $k T_{\mathrm{bb}}$, in the energy range of a few $\mathrm{keV}$. The energy-dependent decay time of these bursts is attributed to the cooling of the NS photosphere resulting in a gradual softening of the burst spectrum. For a review, see, e.g. Lewin et al. (1993); Strohmayer \& Bildsten (2006). The time-resolved spectral analysis of the burst was carried out by using BAT and XRT data in the 15-35 keV and 0.3-10 keV bands, respectively. Burst spectra were described reliably by a simple photoelectrically-absorbed black-body, BB, model. For all these spectral fits, the $N_{\mathrm{H}}$ value was frozen at $3.1 \times 10^{22} \mathrm{~cm}^{-2}$ derived from the pre-burst persistent emission (see Table 1). The inferred BB temperature, $k T_{\mathrm{bb}}$, apparent BB

\footnotetext{
3 The rise time is defined as the time spent between the start of the burst and the point at which the $90 \%$ of the peak burst intensity is reached.
} 


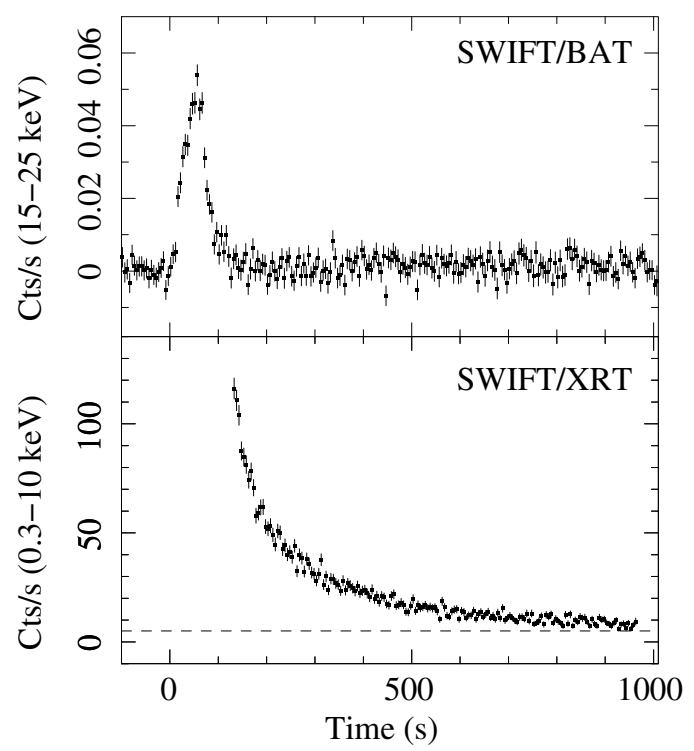

Fig. 3. The intermediate, long, type-I X-ray bursts detected from XTE J1701-407 on July 17, 2008. The time $T_{0}$ expressed in UTC corresponds to $17^{\mathrm{h}} 31^{\mathrm{m}} 55^{\mathrm{s}}$. The Swift/BAT (15-25 keV) and XRT $(0.3-10 \mathrm{keV})$ light curve are shown with a time bin of $5 \mathrm{~s}$. The data gap of $\approx 133 \mathrm{~s}$ in the XRT light curve is due to the time elapsed between the BAT trigger and the follow-up observation. The dashed line indicates the background level measured $\approx 3000 \mathrm{~s}$ after the burst end.

radius at $6.2 \mathrm{kpc}$ (see Sect. 3.1), $R_{\mathrm{bb}}$, and bolometric luminosity are shown in Fig. 5 and in Table 3. The burst fluence is calculated from bolometric fluxes, $F_{\text {bol }}$; these correspond to the observed 2-10 keV Swift/XTR fluxes extrapolated to the 0.1$100 \mathrm{keV}$ energy range. The peak flux, $F_{\text {peak }}$, is derived from the BAT $15-35 \mathrm{keV}$ light curve spectra and extrapolated to the $0.1-$ $100 \mathrm{keV}$ energy range. All the measured unabsorbed fluxes were extrapolated to the $0.1-100 \mathrm{keV}$ band by generating dummy responses (XSPEC version 11.3.2ag). This is justifiable for the XRT data since the black-body temperature is well inside the spectra bandpass. However, during the main peak a black-body temperature reaches a maximun in the energy range $2-3 \mathrm{keV}$ (see e.g., Falanga et al. 2008, and references therein), and therefore extrapolating the black-body spectra outside the BAT bandpass. For the best-fit black-body model to the BAT (15-35 keV) burst peak spectrum, we found $k T_{\mathrm{bb}}=2.68 \pm 0.21\left(\chi_{\text {red }}^{2}=0.74,7\right.$ d.o.f.). We fixed an upper and lower boundary black-body temperature of 2 and $3 \mathrm{keV}$, respectively, the normalization remaining as a free parameter. Note the lower boundary is also consistent to be higher as the first XRT black-body temperature of $k T_{\mathrm{bb}} \approx 1.8$ (see Table 3 ). We found an unacceptable fit by using a black-body function of $2 \mathrm{keV}$ burst peak temperature $\left(\chi_{\text {red }}^{2}=5.1\right.$, 8 d.o.f.), whereas a slightly more reliable fit was obtained by using a temperature of $3 \mathrm{keV}\left(\chi_{\text {red }}^{2}=1.22,8\right.$ d.o.f. $)$. In Fig. 4, we show the BAT data with the different black-body models corresponding to the different fits. In this case, the best-fit model parameters and the extrapolated peak flux are acceptable values to an accuracy of within $20 \%$, which is inside our BAT extrapolation error box. In Table 2, we report all the measured burst parameters.

From the time-resolved spectral results, we converted the light-curve count rates to flux and also found in this case that a double-exponential function was required to fit the intermediate long-burst decay. The derived e-folding times are $\tau_{1} \approx 40 \pm 3 \mathrm{~s}$ and $\tau_{2} \approx 221 \pm 9 \mathrm{~s}$, respectively with $\chi_{\text {red }}^{2}=1.1$ (for 163 d.o.f.).

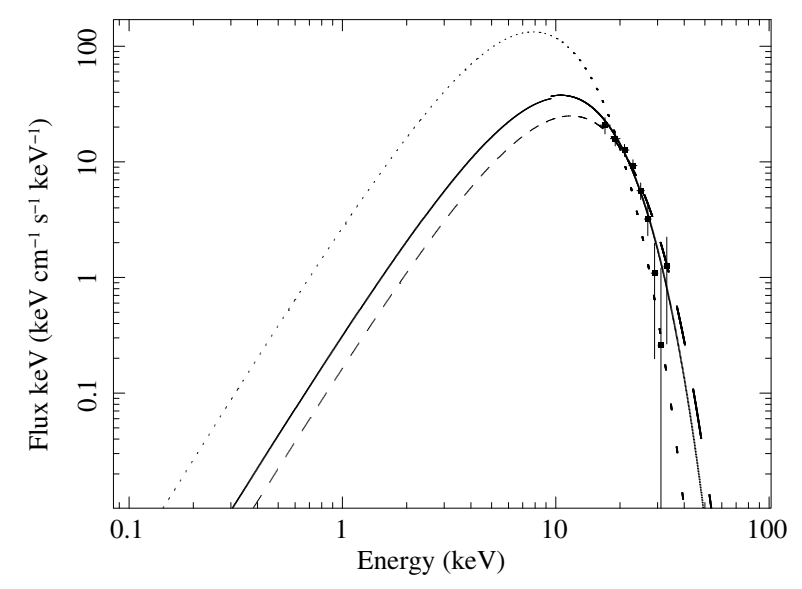

Fig. 4. Swift/BAT (15-35 keV) data of the intermediate long peak blackbody spectra and best-fit model, $k T_{\mathrm{bb}} \approx 2.7$, (solid line). Upper limit, $k T_{\mathrm{bb}}=3 \mathrm{keV}$, acceptable fit (dashed-line) and lower, $k T_{\mathrm{bb}}=2 \mathrm{keV}$, unacceptable fit (dotted-line).

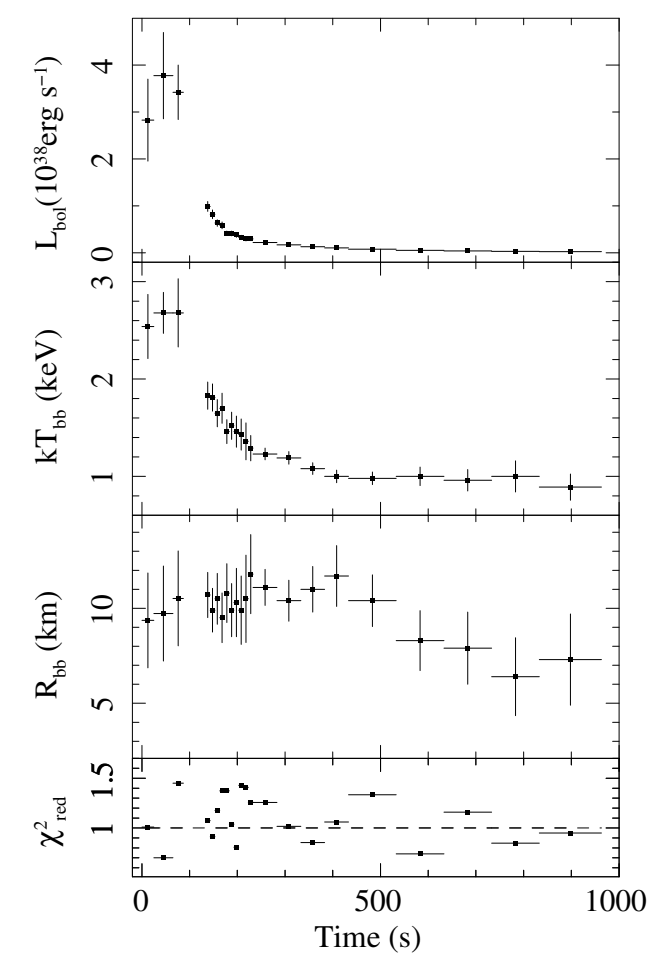

Fig. 5. Evolution of the spectral parameters, as inferred from Swift/BAT (first $86 \mathrm{~s}$ ) and Swift/XRT (833 s) observations. The bolometric luminosity is calculated by assuming a distance of $6.2 \mathrm{kpc}$, see Sect. 3.1. The bottom panel shows the $\chi_{\text {red }}^{2}$ values for these fits.

In this fit, we also included the first two BAT data points. We note, using only the XRT data points, we found the same results within the error bars. A single exponential function was found to be inadequate with $\chi_{\text {red }}^{2}=5.2$ (166 d.o.f.). To compare the decay tail with the intermediate long $\mathrm{X}$-ray burst from 2S 0918-549 and SLX 1737-282 (in 't Zand et al. 2005; Falanga et al. 2008), and with the decay cooling model from Cumming \& Macbeth (2004), we fitted the data with a power-law, and found an index of $\Gamma=-2.14 \pm 0.02$ with a $\chi_{\text {red }}^{2}=1.7$ (167 d.o.f.). In Fig. 6, we show the double-exponential best-fit model (upper panel) and, in a log-log scale, the power-law best-fit function (lower panel) to the data. The double-exponential model fit is 
Table 2. Parameters of the Intermediate long-burst.

\begin{tabular}{ll}
\hline \hline$F_{\text {peak }}^{a}\left(\mathrm{erg} \mathrm{cm}^{-2} \mathrm{~s}^{-1}\right)$ & $8.2 \pm 2 \times 10^{-8}$ \\
$f_{\mathrm{b}}^{b}\left(\mathrm{erg} \mathrm{cm}^{-2}\right)$ & $7.6 \pm 0.3 \times 10^{-6}$ \\
$\tau \equiv f_{\mathrm{b}} / F_{\text {peak }}(\mathrm{s})$ & $92 \pm 22$ \\
$\gamma \equiv F_{\text {pers }}^{c} / F_{\text {peak }}$ & $2.2 \pm 0.5 \times 10^{-2}$ \\
\hline
\end{tabular}

${ }^{a}$ Unabsorbed flux $(0.1-100 \mathrm{keV}) .{ }^{b}$ Net burst fluence $(0.1-100 \mathrm{keV})$. ${ }^{c}$ Unabsorbed persistent flux $F_{\text {pers }}=(1.8 \pm 0.2) \times 10^{-9} \mathrm{erg} \mathrm{cm}^{-2} \mathrm{~s}^{-1}$ $(0.1-100 \mathrm{keV})$.
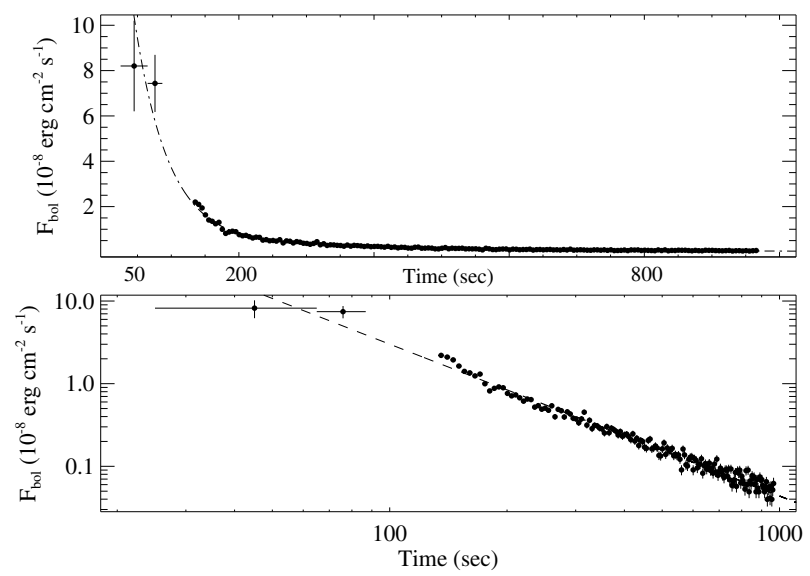

Fig. 6. Top panel: we show the BAT and XRT intermediate longburst light curve with the double-exponential best-fit model curve. Bottom panel: the same data presented in a log-log scale with the powerlaw best-fit model.

statistically preferred over an power-law fit by the F-test probability of $1.2 \times 10^{-14}$. Similar values of $\tau_{1}=44 \pm 7 \mathrm{~s}$ and $\tau_{2}=218 \pm 55 \mathrm{~s}$ or power-law index $\Gamma=-1.97 \pm 0.09$ can be obtained from the fit to the bolometric luminosity reported in Fig. 5. In this case the double-exponential function fit is also statistically preferred over a power-law function fit by the F-test probability of $6.6 \times 10^{-3}$.

The decay time determined with the light curve in unit of count rates is restricted to the XRT $0.3-10 \mathrm{keV}$ energy band. This decay time does not take account of the different rise and cooling effects for different energy bands during the outburst, where by converting the rates to bolometric flux or luminosity using the time-resolved spectral results, a more realistic decay time can be obtained. Therefore, in the following we consider only $\tau_{1} \approx 40 \mathrm{~s}$ and $\tau_{2} \approx 221 \mathrm{~s}$, or the power-law index -2.14 . In Table 2, we report the $\tau \equiv f_{\mathrm{b}} / F_{\text {peak }}=92 \pm 22 \mathrm{~s}$, which is valid only if one exponential function describes the decay tail. In our case, this value is consistent within the error bars if we consider the double exponential decay to be $f_{\mathrm{b}} / F_{\text {peak }}=\epsilon\left(\tau_{1}-\tau_{2}\right)+\tau_{2}=$ $71 \pm 12$, where $\epsilon=F_{x} / F_{\text {peak }}=0.83$ and $F_{x}$ is the peak flux for $\tau_{1}$.

\subsection{Short-burst light curves and spectra}

In Fig. 7, we show the Swift/BAT 15-25 keV short-burst light curve. The time $T_{0}$ expressed in UTC corresponds to $22^{\mathrm{h}} 31^{\mathrm{m}} 19^{\mathrm{s}}$. The rise time is $2 \pm 0.5 \mathrm{~s}$, where the total duration is $\approx 8 \mathrm{~s}$. To determine the rise time and burst duration, we rebinned the light curve into bins of $1 \mathrm{~s}$. For this short burst, the XRT light curve starts with a delay of $180 \mathrm{~s}$ after the beginning of the burst as seen by BAT. In the XRT data $(0.3-10 \mathrm{keV})$, the burst was not detected, so the total duration of this burst must be considered
Table 3. Fit parameters of the time-averaged BAT and XRT burst spectra.

\begin{tabular}{ccccc}
\hline \hline $\begin{array}{c}\Delta \mathrm{T} \\
(\mathrm{s})\end{array}$ & $\begin{array}{c}L_{\mathrm{bol}} \\
\left(10^{38} \mathrm{erg} \mathrm{s}^{-1}\right)\end{array}$ & $\begin{array}{c}k T_{\mathrm{bb}} \\
(\mathrm{keV})\end{array}$ & $\begin{array}{c}R_{\mathrm{bb}} \\
(\mathrm{km})\end{array}$ & $\begin{array}{c}\chi^{2} / \text { d.o.f. } \\
-\end{array}$ \\
\hline $25^{a}$ & $2.8 \pm 0.8$ & $2.54 \pm 0.33$ & $9.3 \pm 2.5$ & $6.9 / 7$ \\
$40^{a}$ & $3.8 \pm 0.9$ & $2.68 \pm 0.21$ & $9.7 \pm 2.5$ & $4.9 / 7$ \\
$22^{a}$ & $3.4 \pm 0.6$ & $2.68 \pm 0.35$ & $10.5 \pm 2.5$ & $10.1 / 7$ \\
10 & $1.0 \pm 0.1$ & $1.83 \pm 0.14$ & $10.7 \pm 1.2$ & $54.8 / 51$ \\
10 & $0.82 \pm 0.09$ & $1.81 \pm 0.14$ & $9.9 \pm 1.1$ & $39.2 / 43$ \\
10 & $0.63 \pm 0.07$ & $1.65 \pm 0.14$ & $10.5 \pm 1.3$ & $43.4 / 37$ \\
10 & $0.58 \pm 0.07$ & $1.70 \pm 0.15$ & $9.5 \pm 1.3$ & $45.3 / 33$ \\
10 & $0.42 \pm 0.05$ & $1.46 \pm 0.12$ & $10.8 \pm 1.5$ & $38.5 / 28$ \\
10 & $0.41 \pm 0.05$ & $1.52 \pm 0.14$ & $9.9 \pm 1.4$ & $27.0 / 26$ \\
10 & $0.38 \pm 0.05$ & $1.46 \pm 0.16$ & $10.3 \pm 1.8$ & $19.4 / 24$ \\
10 & $0.33 \pm 0.05$ & $1.43 \pm 0.16$ & $9.9 \pm 1.8$ & $33.4 / 22$ \\
10 & $0.29 \pm 0.04$ & $1.36 \pm 0.19$ & $10.5 \pm 2.3$ & $26.7 / 19$ \\
10 & $0.30 \pm 0.04$ & $1.29 \pm 0.13$ & $11.8 \pm 2.1$ & $26.4 / 21$ \\
50 & $0.22 \pm 0.01$ & $1.23 \pm 0.06$ & $11.1 \pm 0.9$ & $109.4 / 87$ \\
50 & $0.17 \pm 0.01$ & $1.19 \pm 0.06$ & $10.4 \pm 1.1$ & $70.7 / 70$ \\
50 & $0.13 \pm 0.01$ & $1.08 \pm 0.06$ & $11.0 \pm 1.2$ & $50.3 / 59$ \\
50 & $0.11 \pm 0.01$ & $1.00 \pm 0.06$ & $11.7 \pm 1.6$ & $53.0 / 50$ \\
100 & $0.077 \pm 0.009$ & $0.98 \pm 0.06$ & $10.4 \pm 1.4$ & $100.1 / 75$ \\
100 & $0.054 \pm 0.007$ & $1.00 \pm 0.09$ & $8.3 \pm 1.6$ & $45.1 / 61$ \\
100 & $0.042 \pm 0.007$ & $0.96 \pm 0.11$ & $7.9 \pm 1.9$ & $60.3 / 52$ \\
100 & $0.033 \pm 0.009$ & $1.00 \pm 0.16$ & $6.4 \pm 2.0$ & $38.2 / 45$ \\
130 & $0.026 \pm 0.009$ & $0.89 \pm 0.14$ & $7.3 \pm 2.4$ & $49.4 / 52$ \\
\hline
\end{tabular}

${ }^{a}$ BAT spectrum.

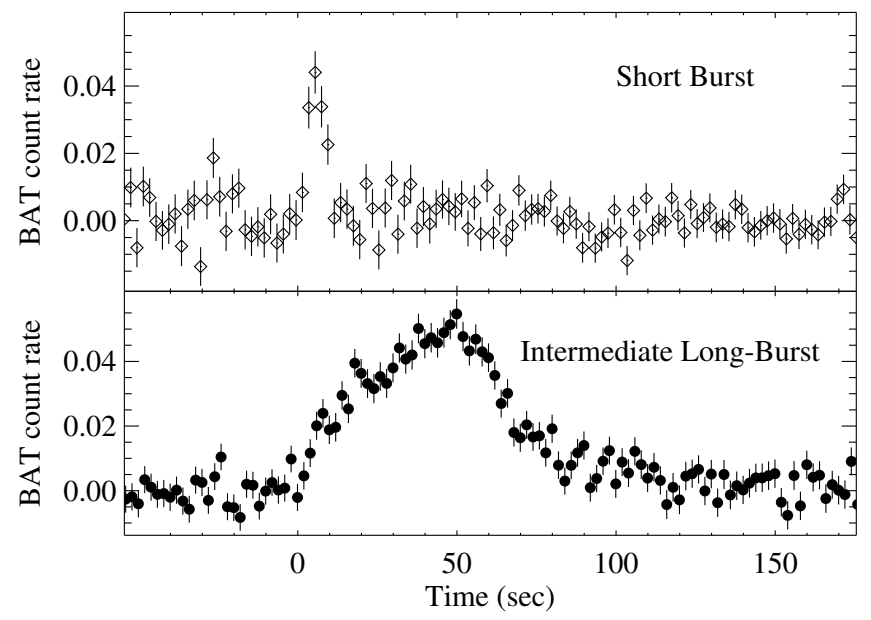

Fig. 7. Top panel: the short type-I X-ray burst detected from XTE J1701-407 on July 27, 2008. For comparison of the rise time and duration we show also the intermediate long-burst (see also Fig. 3). The Swift/BAT (15-25 keV) light curves are shown with a time bin of $2 \mathrm{~s}$.

$<180$ s. In Fig. 7, we show the short burst, and for comparison we also plot the intermediate long-burst observed by BAT.

Given the short duration and statistic of the burst light curve, we were unable to study a time-resolved spectral analysis. Here, we report the burst spectrum analysis integrated over intervals of $6 \mathrm{~s}$ in order to be able to measure as accurately as possible the peak flux. Burst spectra were well fit by a simple black-body model. The inferred BB temperature, $k T_{\mathrm{bb}}=3.9 \pm 0.5 \mathrm{keV}$ with an unabsorbed, bolometric peak fluxes, $F_{\text {bol }}=3.8 \pm 1.1 \times 10^{-8}$ $(0.1-100 \mathrm{keV})$. The burst occurred at a flux persistent level of $2.3 \pm 0.4 \times 10^{-9} \mathrm{erg} \mathrm{cm}^{-2} \mathrm{~s}^{-1}$. This short burst occurred at a persistent emission level comparable to that of the intermediate long-burst. 


\section{Discussion}

\subsection{Source distance, persistent flux, and accretion rate}

When a burst undergoes a photospheric-radius expansion (PRE), the source distance can be determined based on the assumption that the bolometric peak luminosity is saturated at the Eddington limit, $L_{\text {Edd }}$, (e.g., Lewin et al. 1993; Kuulkers et al. 2003). During the PRE episode, while the bolometric luminosity remains constant at the Eddington value, the high energy flux may display a double-peak profile and/or a delay in the rise time (e.g., Kuulkers et al. 2002; Galloway et al. 2006a; Falanga et al. 2007). The BAT light curve shows a slow rise time of $\approx 45 \mathrm{~s}$, which is typically observed at high energy in intermediate long helium bursts with PRE (e.g., Kuulkers et al. 2002; Molkov et al. 2005; Falanga et al. 2008).

Since Swift/XRT missed the first 133 seconds of the burst from XTE J1701-407, we are unable to determine directly whether this burst experienced a PRE. This issue cannot be resolved with BAT time-resolved spectral analysis due to limited statistics (see Fig. 5). However, by comparing with other PRE bursts exhibiting this slow rise time at high energy, we infer that the observed profile in the BAT light curve is evidence of a PRE during the first $\approx 50 \mathrm{~s}$ of the intermediate long-burst, which should correspond to the timescale for the photosphere to fall back to the neutron star surface. For the short burst, no conclusion can be drawn about whether a PRE event has occurred (see Fig. 7).

Another possibility is that the burst has an intrinsically long rise time. For example, the mixed $\mathrm{H} / \mathrm{He}$ bursts observed from GS 1826-24 (Galloway et al. 2004) do not exhibit PRE, but have a rise lasting $10 \mathrm{~s}$, constrained by hydrogen burning (Heger et al. 2007). The duration of the bursts from GS 1826-24 is far shorter than the long-burst from XTE J1701-407. It is unclear whether a long-duration, mixed $\mathrm{H} / \mathrm{He}$ burst could have a $50 \mathrm{~s}$ rise (see discussion of light curves in Sect. 3.5). The fact that helium burning can be extremely rapid, whereas hydrogen burning involves slow, weak interactions means that the rise time is longer when hydrogen is present, and PRE is far less likely. For example, Fujimoto et al. (1987) derived a critical, helium fraction that is necessary to achieve PRE.

Assuming a bolometric peak luminosity equal to the Eddington value for a He type-I X-ray burst $\left(L_{\text {Edd }} \approx 3.8 \times\right.$ $10^{38} \mathrm{erg}^{-1}$, as empirically derived by Kuulkers et al. 2003), we obtain the source distance of $d=6.2_{-0.9}^{+1.6} \mathrm{kpc}$. For comparison, the theoretical value of this distance (e.g., Lewin et al. 1993) found by assuming a He atmosphere and canonical NS parameters $(1.4$ solar mass and radius of $10 \mathrm{~km})$, is $5.5_{-0.8}^{+1.3} \mathrm{kpc}$. We note that the source could be closer if the peak luminosity of the burst was lower than the pure-helium, Eddington limit. For example, we cannot exclude the possibility of the burst not exhibiting PRE, in which case the peak luminosity could have been subEddington. Alternatively, assuming the peak luminosity to be the Eddington luminosity for solar composition $\left(X_{0}=0.7\right)$ infers a distance of $\approx 4 \mathrm{kpc}$. In the following, we consider $d \approx 6.2 \mathrm{kpc}$ to be a fiducial distance, and comment on how our conclusions would change if the source was closer.

The best-fit model solution of the $2-20 \mathrm{keV}$ persistent emission spectrum of XTE J1701-407 is an absorbed, simple, powerlaw model with $\Gamma \approx 2.1$. Assuming a distance of $6.2 \mathrm{kpc}$, the estimated intermediate long-burst, pre-burst persistent unabsorbed flux between $0.1-100 \mathrm{keV}, F_{\text {pers }} \approx 1.9 \times 10^{-9} \mathrm{erg} \mathrm{cm}^{-2} \mathrm{~s}^{-1}$, translates into a bolometric luminosity of $L_{\text {pers }} \approx 8.3 \times 10^{36} \mathrm{erg} \mathrm{s}^{-1}$, or $\approx 2.2 \% L_{\text {Edd }}$.
The local accretion rate per unit area is then given by $L_{\mathrm{pers}}=$ $4 \pi R^{2} \dot{m}(G M / R) /(1+z)$, or

$$
\begin{aligned}
\dot{m}= & 4.0 \times 10^{3} \mathrm{~g} \mathrm{~cm}^{-2} \mathrm{~s}^{-1} \\
& \times\left(\frac{R}{11.2 \mathrm{~km}}\right)^{-1}\left(\frac{M}{1.4 M_{\odot}}\right)^{-1}\left(\frac{d}{6.2 \mathrm{kpc}}\right)^{2}\left(\frac{1+z}{1.26}\right) .
\end{aligned}
$$

A convenient unit of accretion rate is the Eddington accretion rate. Here, we define the local Eddington accretion rate to be $\dot{m}_{\text {Edd }} \equiv 1.8 \times 10^{5} \mathrm{~g} \mathrm{~cm}^{-2} \mathrm{~s}^{-1}$, which corresponds to the local accretion rate onto a neutron star of mass $M=1.4 M_{\odot}$, and radius $R=11.2 \mathrm{~km}$ of accretion luminosity equal to the Eddington luminosity $3.8 \times 10^{38} \mathrm{erg} \mathrm{s}^{-1}$ empirically derived by Kuulkers et al. (2003). This infers that $\dot{m} / \dot{m}_{\text {Edd }}=2.2 \%$.

\subsection{The energy, ignition depth, and recurrence time of the long-burst}

The observed energy of the long-burst allows us to estimate the ignition depth. The measured fluence of the burst is $f_{\mathrm{b}}=7.6 \times$ $10^{-6} \mathrm{erg} \mathrm{cm}^{-2}$, corresponding to a net burst energy release of $E_{\text {burst }}=4 \pi d^{2} f_{\mathrm{b}}=3.5 \times 10^{40} \mathrm{erg}(d / 6.2 \mathrm{kpc})^{2}$. The ignition depth is given by $E_{\text {burst }}=4 \pi R^{2} y_{\text {ign }} Q_{\text {nuc }} /(1+z)$, or

$$
\begin{aligned}
y_{\text {ign }}= & 1.8 \times 10^{9} \mathrm{~g} \mathrm{~cm}^{-2}\left(\frac{d}{6.2 \mathrm{kpc}}\right)^{2} \\
& \times\left(\frac{Q_{\text {nuc }}}{1.6 \mathrm{MeV} / \text { nucleon }}\right)^{-1}\left(\frac{R}{11.2 \mathrm{~km}}\right)^{-2}\left(\frac{1+z}{1.26}\right) .
\end{aligned}
$$

The value of $Q_{\text {nuc }} \approx 1.6 \mathrm{MeV}$ corresponds to the nuclear-energy release per nucleon for complete burning of helium to iron group elements. Including hydrogen with a mass-weighted mean mass fraction $\langle X\rangle$ provides a value of $Q_{\mathrm{nuc}} \approx 1.6+4\langle X\rangle \mathrm{MeV} /$ nucleon (Galloway et al. 2004), where we include losses due to neutrino emission following Fujimoto et al. (1987). For $\langle X\rangle=0.7$, the solar composition value, $Q_{\text {nuc }}=4.4 \mathrm{MeV} /$ nucleon, and $y_{\text {ign }}=$ $6.5 \times 10^{8} \mathrm{~g} \mathrm{~cm}^{-2}$.

At an accretion rate of $4 \times 10^{3} \mathrm{~g} \mathrm{~cm}^{-2} \mathrm{~s}^{-1}$, the recurrence time corresponding to a column depth of $y_{\text {ign }}=1.8 \times 10^{9} \mathrm{~g} \mathrm{~cm}^{-2}$ (pure helium composition) is $\Delta t=\left(y_{\text {ign }} / \dot{m}\right)(1+z)=6.6$ days, or for $y_{\text {ign }}=6.5 \times 10^{8} \mathrm{~g} \mathrm{~cm}^{-2}$ (solar composition) is $\Delta t=$ 2.4 days. These derived recurrence times are independent of the assumed distance. The intermediate long-burst, which was the first observed burst, occurred 40 days after the detection of XTE J1701-407. The effective exposure time on source from the beginning of the outburst to the intermediate long-burst was 0.34 days, and there were 0.14 days between both bursts. The elapsed time on source was thus too short compared to the theoretically-derived recurrence time to allow us to derive an observational measurement of the recurrence time.

\subsection{Theoretical comparison with ignition models}

In attempting to understand the nuclear-burning processes responsible for the long-burst, we compare the observed properties with type-I X-ray burst-ignition models. The ignition conditions were calculated as described by Cumming \& Bildsten (2000), apart from assuming that $R=11.2 \mathrm{~km}, M=1.4 M_{\odot}$, and the energy release in hot $\mathrm{CNO}$ burning ${ }^{4} E_{\mathrm{H}}=6.0 \times 10^{18} \mathrm{erg} \mathrm{g}^{-1}$. The

\footnotetext{
${ }^{4}$ Here we adopt the value of $E_{\mathrm{H}}$ from Wallace \& Woosley (1981), which includes neutrino losses. These were not included by Cumming \& Bildsten (2000).
} 
Table 4. Type-I X-ray burst-ignition conditions ${ }^{a}$.

\begin{tabular}{lllllllllllll}
\hline \hline Model & $\begin{array}{l}\dot{m}^{b} \\
\left(\% \dot{m}_{\text {Edd }}\right)\end{array}$ & $Z_{\mathrm{CNO}}$ & $X_{0}^{c}$ & $Q_{\mathrm{b}}$ & $y_{\mathrm{ign}, 9}$ & $T_{\mathrm{ign}, 8}$ & $\langle X\rangle$ & $X_{\mathrm{b}}$ & $Q_{\text {nuc }}$ & $E_{40}$ & $\begin{array}{l}\Delta t \\
\text { (days) }\end{array}$ \\
\hline \multicolumn{10}{c}{ Pure helium accretion } \\
1 & 2.2 & 0.02 & 0 & 0.5 & 1.8 & 1.4 & 0 & 0 & 1.6 & 3.4 & 6.5 \\
2 & 2.2 & 0.02 & 0 & 0.3 & 7.4 & 1.2 & 0 & 0 & 1.6 & 14 & 27 \\
3 & 2.2 & 0.02 & 0 & 0.7 & 0.84 & 1.5 & 0 & 0 & 1.6 & 1.6 & 3.1 \\
\hline \multicolumn{10}{c}{ Accretion hydrogen-rich material } \\
4 & 2.2 & 0.02 & 0.7 & 0.5 & 0.13 & 2.0 & 0.38 & 0.07 & 3.1 & 0.49 & 0.48 \\
5 & 0.69 & 0.02 & 0.7 & 0.5 & 1.6 & 1.4 & 0.01 & 0 & 1.64 & 3.2 & 19 \\
6 & 2.2 & 0.001 & 0.7 & 0.1 & 0.67 & 1.7 & 0.62 & 0.54 & 4.1 & 3.3 & 2.5 \\
7 & 2.2 & 0.001 & 0.7 & 0.5 & 0.53 & 1.8 & 0.63 & 0.43 & 4.1 & 2.7 & 2.0 \\
\hline
\end{tabular}

${ }^{a}$ Models 1, 5, and 6 provide a good match to the observed burst energy of $3.5 \times 10^{40} \mathrm{erg}$. In addition, models 1 and 6 have an accretion rate that matches the value inferred from the persistent luminosity; ${ }^{b}$ we define $\dot{m}_{\mathrm{Edd}}=1.8 \times 10^{5} \mathrm{~g} \mathrm{~cm}^{-2} \mathrm{~s}^{-1}$, the local accretion rate onto a $1.4 M_{\odot}$, $R=11.2 \mathrm{~km}$ neutron star, which infers an accretion luminosity equal to the empirically-derived Eddington luminosity $3.8 \times 10^{38} \mathrm{erg} \mathrm{s}^{-1}$ from Kuulkers et al. (2003); ${ }^{c}$ the hydrogen mass fractions are: in the accreted material $X_{0}$, at the base of the layer at ignition $X_{\mathrm{b}}$, and the mass-weighted mean value in the layer at ignition $\langle X\rangle ;{ }^{d}$ note that the ignition conditions for pure helium accretion do not depend on the choice of $Z_{\mathrm{CNO}}$.

results are shown in Table 4 for several different choices of accreted hydrogen fraction $X_{0}$, accretion rate $\dot{m}$, and flux from the crust $Q_{\mathrm{b}}$. The ignition conditions are calculated by modeling the temperature profile of the accumulated fuel layer, and adjusting the thickness of the layer until conditions for thermal runaway are achieved at the base. These models do not include the effects of previous bursts on the ignition conditions (e.g. Woosley et al. 2004), and we do not include gravitational sedimentation, which is important at low accretion rates (Peng et al. 2007). In the following, we consider three possibilities: 1) accretion of pure helium; 2) hydrogen-rich matter at solar; and 3) low metallicity.

\subsubsection{Pure helium accretion}

For pure helium accretion, the accumulating fuel layer is heated from below by a flux $\dot{m} Q_{\mathrm{b}}$ originating in the crust, where $Q_{\mathrm{b}}$ is the energy per accreted nucleon flowing outwards from the crust. We assume the accretion rate to be the observed value $\dot{m} / \dot{m}_{\text {Edd }}=2.2 \%$, and adjust the value of $Q_{\mathrm{b}}$ until we achieve ignition at the inferred column for pure helium burning $y_{\text {ign }}=$ $1.8 \times 10^{9} \mathrm{~g} \mathrm{~cm}^{-2}$ (and therefore obtain the observed burst energy). A flux from below $Q_{\mathrm{b}}=0.5 \mathrm{MeV} /$ nucleon reproduces the observed burst energy at $2.2 \%$ Eddington. We also include models with $Q_{\mathrm{b}}=0.3$ and $0.7 \mathrm{MeV} /$ nucleon in Table 4 to illustrate the sensitivity of the ignition depth to the amount of heat from below. We note that since the combination $\dot{m} Q_{\mathrm{b}}$ defines the total flux heating the layer, an increase or decrease in $Q_{\mathrm{b}}$ while keeping $\dot{m}$ fixed is equivalent to increasing or decreasing $\dot{m}$ with $Q_{\mathrm{b}}$ fixed. Therefore, if the inferred accretion rate is lower by some factor, the value of $Q_{\mathrm{b}}$ needed to match the observed energy will be higher by the same factor. Similarly, a source distance of less than $6.2 \mathrm{kpc}$ can be described accurately by increasing $Q_{\mathrm{b}}$.

The outward flux expected from the crust depends on the accretion rate and the core neutrino emissivity (Brown 2000), which ranges from $\dot{m} Q_{\mathrm{b}} \approx 0.1 \mathrm{MeV}$ per nucleon at high accretion rates to $\dot{m} Q_{\mathrm{b}} \approx 1.5 \mathrm{MeV}$ per nucleon at low accretion rates. The value depends on how much of the total $\approx 1.5 \mathrm{MeV}$ per nucleon heat released in the crust by pycnonuclear and electron capture reactions (Haensel \& Zdunik 1990, 2008), is conducted into the core and released as neutrinos, compared to being conducted outwards. Our calculated value of $0.5 \mathrm{MeV} /$ nucleon is reasonable for an accretion rate of $2.2 \%$ Eddington; for example, the models calculated by Cumming et al. (2006) for persistently accreting sources have $Q_{\mathrm{b}}=0.3$ to $0.9 \mathrm{MeV} /$ nucleon at this accretion rate. Galloway \& Cumming (2006b) modelled the Xray bursts observed from SAX J1808.4-3658 and found $Q_{\mathrm{b}} \approx$ $0.3 \mathrm{MeV} /$ nucleon for $\dot{m} \approx 3 \% \dot{m}_{\text {Edd }}$.

\subsubsection{Accretion of hydrogen rich matter with solar metallicity}

We next, consider that the source accretes hydrogen-rich matter with the solar hydrogen fraction $X_{0}=0.7$ and a metallicity similar to solar, with mass fraction of CNO elements $Z_{\mathrm{CNO}}=0.02$. In that case, the hydrogen burns at a fixed rate during accumulation of the fuel layer, by the beta-limited hot CNO cycle of Hoyle \& Fowler (1965). The hydrogen depletes at a column depth of

$y_{\mathrm{d}}=1.5 \times 10^{8} \mathrm{~g} \mathrm{~cm}^{-2}\left(\frac{\dot{m}}{0.022 \dot{m}_{\mathrm{Edd}}}\right)\left(\frac{Z_{\mathrm{CNO}}}{0.02}\right)^{-1}\left(\frac{X_{0}}{0.7}\right)$,

which is lower than the inferred, ignition depth, so that a thick layer of pure helium accumulates and ignites beneath a steady hydrogen-burning shell. Therefore, even though solar composition material is accreted, the mean hydrogen fraction at ignition in the fuel layer is low, implying that $Q_{\text {nuc }}$ is close to the value of $1.6 \mathrm{MeV} /$ nucleon for pure helium.

For a CNO mass fraction of $Z_{\mathrm{CNO}}=0.02$, the observed burst energy is obtained for an accretion rate three times lower than inferred from the observed X-ray luminosity $\dot{m} / \dot{m}_{\text {Edd }}=0.69 \%$ (model 5 in Table 4). At an accretion rate of $2.2 \%$ Eddington, the burst energy is a factor of seven too small (model 4). Reducing the distance to the source does not help because it would change both the inferred $\dot{m}$ and burst energy by the same factor.

\subsubsection{Accretion of hydrogen-rich matter of low metallicity}

In the third scenario, we consider that the material is hydrogenrich, but with a low metallicity. Model 6 has a burst energy close to the observed energy at the inferred accretion rate of $2.2 \%$ Eddington, with a CNO mass fraction $Z=10^{-3}$, approximately $10 \%$ of the solar metallicity. In this case, the amount of hot CNO burning is reduced substantially, so that hydrogen permeates the entire fuel layer at ignition. Hot CNO burning still operates at a low level and causes some preheating of the fuel layer. The hydrogen increases the amount of nuclear release during the burst, giving $Q_{\text {nuc }} \approx 4 \mathrm{MeV} /$ nucleon, and an ignition depth three times smaller than for the pure helium case. The low 
metallicity ignition is less sensitive to $Q_{\mathrm{b}}$ than the pure helium ignition. Model 7, which has the same conditions as model 6, but with $Q_{\mathrm{b}}=0.5$ rather than $Q_{\mathrm{b}}=0.1$, has a burst energy within almost $20 \%$ of the observed value. As for to the pure-helium ignitions, a closer distance can be accommodated by varying $Q_{\mathrm{b}}$.

One caveat regarding the low-metallicity ignition-models is that there can be substantial heating of the accumulating fuel layer because of nuclear reactions associated with the ashes of previous bursts. Woosley et al. (2004) found that the burst behaviour at accretion rates of $\approx 0.1$ Eddington was insensitive to the metallicity of the accreted material due to this effect. However, they found that at lower accretion rates there was good agreement with the ignition models presented here (see Woosley et al. 2004, Table 9).

\subsubsection{Summary of ignition models}

We present three ignition models in Table 4 that reproduce the observations. Models 1 and 6 have the correct burst energy and accretion rate. They correspond to accretion of pure helium (model 1), for which the layer is heated by the outward flux from the neutron star crust, and for accretion of hydrogen-rich material with low metallicity (model 6), for which a low level of hydrogen burning preheats the layer during accumulation, but the hydrogen fraction at ignition is significant and makes a substantial contribution to the burst energetics. Third, model 5 has the correct burst energy, but an accretion rate three times lower than observed. Given the uncertainties in translating the observed X-ray luminosity to accretion rate, it seems worthwhile considering this model further. In this model, the accreted composition is hydrogen-rich with a solar metallicity. This leads to depletion of the hydrogen by the hot CNO cycle and the build-up of a thick layer of pure helium beneath the hydrogen shell.

There is a fourth possibility, which is that the source is accreting hydrogen-rich material, but that the hydrogen burns unstably in a series of short flashes. The helium produced in the short flashes accumulates and creates a pure helium layer that ignites to generate the long burst. This is similar to model 5, but with unstable rather than stable hydrogen burning.

One way of distinguishing between the various possible explanations of the burst energetics would be a recurrence-time measurement, since, as shown in Table 4, the different scenarios predict different recurrence times. This is equivalent to a measurement of the $\alpha$ parameter ${ }^{5}$ for the bursts, which would indicate the fuel type (e.g. $\alpha \approx 40$ for solar hydrogen abundance as found for example in GS 1826-24, Galloway et al. 2004).

While this paper was in preparation, a similar analysis of the intermediate burst from XTE J1701-407 was carried out and reported by Linares et al. (2008b). The bolometric peak flux for the long-burst and therefore the limits on the source distance are consistent with the values that we obtain here. However, the persistent luminosity and burst energy measured by Linares et al. (2008b) are a factor of two lower than our values. These values are used to interpret the long-burst, resulting in an accretion rate of $\dot{m}=1.9 \times 10^{3} \mathrm{~g} \mathrm{~cm}^{-2} \mathrm{~s}^{-1}=1.1 \% \dot{m}_{\text {Edd }}$, and a burst energy of $E_{\mathrm{b}}=1.6 \times 10^{40} \mathrm{erg}$, both a factor of two lower than the values we find in this paper. Repeating the ignition calculations presented earlier for this lower accretion rate, we find that accretion of solar composition material $\left(X_{0}=0.7\right)$ produces a burst energy of $E_{\mathrm{b}}=1.5 \times 10^{40} \mathrm{erg}$ for $Q_{\mathrm{b}}=0.1 \mathrm{MeV} /$ nucleon, and

\footnotetext{
5 The quantity $\alpha$ is defined as the ratio of the total energy emitted in the persistent flux to that emitted in a burst, $\alpha=F_{\text {pers }} \Delta t / f_{\mathrm{b}}$, where $\Delta t$ is the time interval between two bursts.
}

$E_{\mathrm{b}}=1.0 \times 10^{40} \mathrm{erg}$ for $Q_{\mathrm{b}}=0.5 \mathrm{MeV} /$ nucleon. Therefore, accretion of solar composition naturally explains the burst energetics for these values of $E_{\mathrm{b}}$ and $\dot{m}$. For pure helium accretion at this rate, we find that $Q_{\mathrm{b}}=1.5 \mathrm{MeV} /$ nucleon is required to achieve a burst energy of $1.5 \times 10^{40} \mathrm{erg}$ (lower values of $Q_{\mathrm{b}}$ result in a deeper ignition and more energetic burst). Although this is a higher value than expected for this accretion rate, it is within the range of the total energy released in the crust (Haensel \& Zdunik 2008). In addition, since the flux heating the layer is proportional to $\dot{m} Q_{\mathrm{b}}$, the requirements on $Q_{\mathrm{b}}$ can be relaxed if the true accretion rate is higher than assumed here. Therefore, we find that the energetics argument provided by Linares et al. (2008b) against explaining this burst as pure helium is overstated.

\subsection{Constraints from the light curve}

The shape and duration of the light curve offer another way to determine the composition of the fuel that burns during the burst. While no models are available with exactly the same burst energy, the low-accretion rate models from Woosley et al. (2004) are within a factor of two to four in energy and accretion rate, and so we compare these models with the observed light curve. These models assume that $\dot{M}=3.5 \times 10^{-10} M_{\odot} \mathrm{yr}^{-1}$, corresponding to a local accretion rate $\dot{m}=1 \% \dot{m}_{\text {Edd }}$.

First, we compare the observed light curve with burst 2 from model zm of Woosley et al. (2004) (solid curve in Fig. 8). This model describes the accretion of hydrogen-rich $\left(X_{0} \approx 0.7\right)$ material of low metallicity, $Z=10^{-3}$, and so is similar to model 6 in Table 4 . The burst has a total energy release of $2.0 \times 10^{40} \mathrm{erg}$, just less than a factor of two lower than the observed burst. The recurrence time is 3.0 days, and ignition column $y_{\text {ign }}=$ $3.6 \times 10^{8} \mathrm{~g} \mathrm{~cm}^{-2}$. The ignition column is just less than a factor of two smaller than the observed burst. The peak luminosity of this burst is close to the Eddington luminosity for solar composition, suggesting that its distance may be closer than the $6.2 \mathrm{kpc}$ assumed here. A closer distance would ensure that these light curves in closer agreement. The model light curve has a steep decline at late times, which is steeper than the observed decline. An extra factor of two in ignition column would provide the correct burst energy and lengthen the model-burst light curve, bringing it into closer agreement with that of the observed burst. Another point to note is that this burst has a slow rise time, lasting for several seconds, as expected for a low helium mass fraction (Fujimoto et al. 1987).

The dashed curve in Fig. 8 shows burst 3 from model $\mathrm{Zm}$ of Woosley et al. (2004). This model has hydrogen-rich matter with solar metallicity, $Z=0.02$, and so is similar to model 5 of Table 4 . The hydrogen burns away in a thin shell, leaving a pure helium layer that ignites. This burst has a total energy release of $8.0 \times 10^{39} \mathrm{erg}$, a factor of 4 smaller than observed. The recurrence time is 4.5 days, and ignition column depth $5.6 \times 10^{8} \mathrm{~g} \mathrm{~cm}^{-2}$. The burst brightens to close to the pure helium Eddington luminosity. The duration of the burst is shorter than the observed burst by a factor of $\approx 5-10$. The slope of the decay is shallower than the observed slope. The rise time of this burst is short, a fraction of a second, in contrast to the much slower rise of the hydrogen-rich burst (model zm; solid line in Fig. 8).

We also include some cooling models calculated following Cumming \& Macbeth (2004). For a given ignition column, an energy release per gram of $1.6 \mathrm{MeV}$ per nucleon is deposited in the layer, as would be appropriate if the helium burned to irongroup elements at each depth at the start of the burst. We also computed models for a lighter ash and correspondingly smaller 


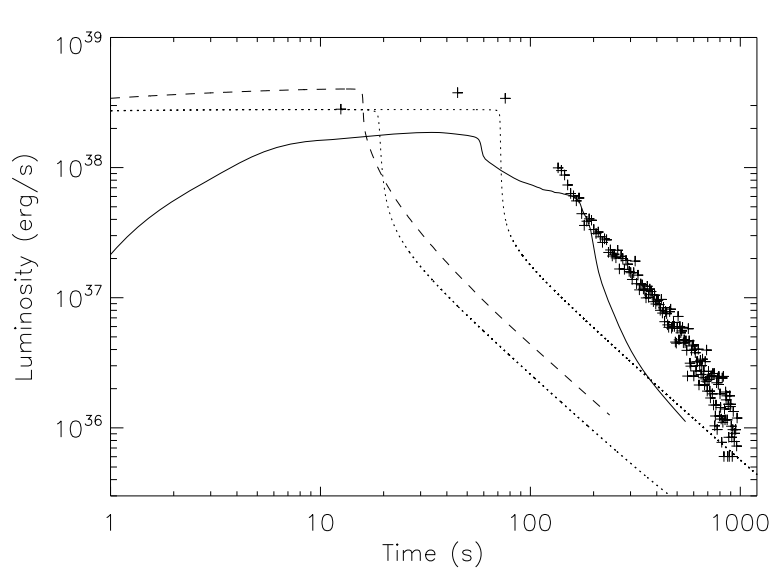

Fig. 8. Model light curves compared with the observed light curve. The solid curve shows burst 2 from model zm of Woosley et al. (2004). This burst has an ignition column and energy a factor of 2 smaller than the observed burst. The dashed curve shows burst 3 from model $\mathrm{Zm}$ of Woosley et al. (2004). This burst has an ignition column a factor of three to four times smaller than the observed burst. The observed bolometric flux has been converted to luminosity using a distance of $6.2 \mathrm{kpc}$. Redshift corrections have been applied to the theoretical light curves, with $1+z=1.26$. The dotted curves show two cooling models calculated following Cumming \& Macbeth (2004), for (left to right) $y_{\text {ign }}=6 \times 10^{8} \mathrm{~g} \mathrm{~cm}^{-2}$ corresponding to Woosley et al. (2004) model $\mathrm{Zm}$ (dashed curve), and $y_{\mathrm{ign}}=2 \times 10^{9} \mathrm{~g} \mathrm{~cm}^{-2}$ as needed to explain the observed burst energy (models 1 and 5 in Table 4).

energy deposition (Woosley et al. (2004) find that the burning does not go all the way to iron group in their model $\mathrm{zM}$ ), but the differences are small, and this does not change our conclusions. The cooling of the layer is then followed, the flux from the surface being limited to the Eddington luminosity for pure helium ${ }^{6}$. We show two examples in Fig. 8. The first has $y_{\text {ign }}=6 \times 10^{8} \mathrm{~g} \mathrm{~cm}^{-2}$ to match the Woosley et al. (2004) model $\mathrm{Zm}$ burst. The second has $y_{\text {ign }}=2 \times 10^{9} \mathrm{~g} \mathrm{~cm}^{-2}$ as needed to reproduce the observed burst energy (models 1 and 5 in Table 4). The shape of the cooling models agrees well with the model $\mathrm{Zm}$ light curve, and agrees to within a factor of two on the cooling timescale. Even allowing for this factor of two in the $y_{\text {ign }}=2 \times 10^{9} \mathrm{~g} \mathrm{~cm}^{-2}$ model, the cooling occurs more rapidly than for the observed light curve.

In summary, although the models from Woosley et al. (2004) are not of exactly the same ignition conditions as implied by the observations of XTE J1701-407, our comparison suggests that pure helium ignition at the inferred ignition column depth (with or without a small overlying hydrogen-burning shell) have cooling times that are shorter than the observed light curve. On the other hand, a hydrogen-rich composition throughout the layer, as expected for low metallicity, produces a longer-lasting light curve that is more consistent with the observed cooling time. This conclusion does not depend on the assumed distance to the source. Both helium and hydrogen-rich burst models reach the Eddington luminosity (either the pure helium or solar composition Eddington luminosity respectively), and therefore could explain the PRE suggested by the similarity between the

\footnotetext{
6 As noted by Woosley et al. (2004), the shape of the light curve as the luminosity begins to decline below the Eddington luminosity is probably not accurately reproduced by these models, which do not follow the outer layers in detail. We will improve our treatment of this in future work. However, we expect that the late time cooling is not sensitive to this.
}

observed BAT light curve and other intermediate long-bursts (see Sect. 3.1). If the slow rise time is intrinsic to the burst and not due to PRE, the hydrogen-rich model is preferred since the presence of hydrogen leads to a far slower rise time than for pure helium (Fig. 8). The double exponential nature of the decay may also argue for hydrogen burning during the burst. The burst profiles from GS 1826-24 (Galloway et al. 2004) are described well by a double exponential decay. Further modeling is required to study the expected burst profiles of hydrogen-rich bursts produced as a result of low-metallicity accretion.

\subsection{Origin of the short burst}

We have focused on the long-duration burst, which has a wellmeasured fluence and therefore energy. For the short burst, we can only place an upper limit on its fluence. At low accretion rates, unstable ignition of hydrogen can produce to shortduration bursts (e.g., Strohmayer \& Bildsten 2006; Chenevez et al. 2007). Linares et al. (2008b) suggested that this is the origin of the short burst from XTE J1701-407, and that either (i) hydrogen-ignited short bursts produce the helium fuel for the long-burst; or that (ii) the source accretes close to the boundary between unstable and stable hydrogen burning, and stable hydrogen-burning produces the helium for the long-burst. The comparison with ignition models and model light curves that we have made earlier suggests that hydrogen survives to the ignition depth, implying a low metallicity in the accreted layer. Unfortunately, this would presumably ensure that a thermal instability driven by CNO burning would be less likely.

For the low-metallicity model or for pure helium accretion, another explanation is that the local accretion rate was higher at the time of the short burst. The ignition depth is sensitive to the base flux or equivalently to the product $\dot{m} Q_{\mathrm{b}}$ (see for example Fig. 8 of in 't Zand et al. 2005). The persistent flux at the time of the short burst from XTE J1701-407 was slightly higher than at the time of the long-burst, even at $30 \%$, insufficient to produce a significant reduction in the ignition column depth. We note that short bursts were observed from the intermediate long X-ray burster 2S 0918-549, a suspected ultracompact binary and therefore accreting hydrogen-deficient matter. Therefore, it is unclear to us that the observation of the short burst excludes pure helium accretion in XTE J1701-407, as argued by Linares et al. (2008b).

\section{Conclusions}

We have compared the observed properties of the long duration burst from XTE J1701-407 with models of type-I X-ray burst-ignition conditions and light curves. We have shown that the observed burst energy could be understood as (i) pure helium ignition, either as a result of pure helium accretion or depletion of hydrogen by steady burning during accumulation; or (ii) ignition of a thick layer of hydrogen-rich material with low metallicity. Comparing with model light-curves, we find that the pure helium ignitions cool faster than observed. On the other hand, a hydrogen-rich layer corresponds to a longer-duration light curve with a steep decline in the tail of the burst, more closely matching the observed light curve. Therefore, we suggest that the intermediate long-burst from XTE J1701-407 was powered by unstable burning of a thick layer of hydrogen-rich matter with low metallicity. Long X-ray bursts caused by pure helium ignitions beneath a hydrogen shell have been identified, as for example by Galloway \& Cumming (2006b), who argued that this occurred in SAX J1808.4-3658. However, to our knowledge, XTE J1701-407 would be the first example of a source 
that exhibits long-bursts driven by a thick layer of hydrogen-rich material. The bursts from GS 1826-24 are believed to be powered by rp-process hydrogen burning, giving long $\approx 100 \mathrm{~s}$ tails, but the ignition depth in those bursts is an order of magnitude smaller than inferred for the long-burst from XTE J1701-407, so that hydrogen can survive until helium ignition, even for solar metallicity. At the low accretion rate in XTE J1701-407, this is not the case: low metallicity is required to reduce the rate of hot CNO burning and allow hydrogen to survive until helium ignites. This implies either that this source is a burster accreting lowmetallicity H-rich material at a low rate, or another possibility is that heavy elements are able to sediment out from the accumulating layer at this low accretion rate (Peng et al. 2007), reducing its effective metallicity. If so, future studies of this source could be used to test the physics of sedimentation at low accretion rates.

Acknowledgements. We thank Alexander Heger for providing the burst light curves from Woosley et al. (2004) shown in Fig. 7. M.F. acknowledges the French Space Agency (CNES) for financial support. J.C. acknowledges financial support from ESA-PRODEX, Nr. 90057, and E.B. acknowledges ASI and MIUR. A.C. acknowledges support from the National Sciences and Engineering Research Council of Canada (NSERC), Le Fonds Québécois de la Recherche sur la Nature et les Technologies (FQRNT), and the Canadian Institute for Advanced Research (CIFAR). A.C. is an Alfred P. Sloan Research Fellow.

\section{References}

Barthelmy, S. D., et al. 2008, GCN Circ., 7985

Brown, E. F. 2000, ApJ, 531, 988

Chelovekov, I. V., Grebenev, S. A., \& Sunyaev, R. A. 2006, AstL, 32, 456

Chenevez, J., Falanga, M., Brandt, S., et al. 2006, A\&A, 449, L5

Chenevez, J., Falanga, M., Kuulkers, E., et al. 2007, A\&A, 469, L27

Cornelisse, R., in 't Zand, J. J. M., Verbunt, F., et al. 2003, A\&A, 405, 1033

Cumming, A., \& Bildsten, L. 2000, ApJ, 544, 453

Cumming, A., \& Bildsten, L. 2001, ApJ, 559, L127

Cumming, A., \& Macbeth, J. 2004, ApJ, 603, L37

Cumming, A., Macbeth, J., in 't Zand, J. J. M., Page, D. 2006, ApJ, 646, 429

Degenaar, N., \& Wijnands, R. 2008, Astr. Tel., 1572
Falanga, M., Poutanen, J., Bonning, E. W., et al. 2007, A\&A, 464, 1069 Falanga, M., Chenevez, J., Cumming, A., et al. 2008, A\&A, 484, 43 Fujimoto, M. Y., Sztajno, M., Lewin, W. H. G., \& van Paradijs, J. 1987, ApJ, 319,902

Galloway, D. K., Cumming, A., Kuulkers, E., et al. 2004, ApJ, 601, 466

Galloway, D. K., Muno, M. P., Hartman, J. M., et al. 2006a, ApJS, submitted, [arXiv: astro-ph/0608259]

Galloway, D. K., \& Cumming, A. 2006b, ApJ, 652, 559

Hansen, C. J., \& Van Horn, H. M. 1975, ApJ, 195, 735

Haensel, P., \& Zdunik, J. L. 1990, A\&A, 227, 431

Haensel, P., \& Zdunik, J. L. 2008, A\&A, 480, 459

Hasinger, G., \& van der Klis, M. 1989, A\&A, 225, 79

Heger, A., Cumming, A., Galloway, D. K., \& Woosley, S. E. 2007, ApJ, 671, L141

Hoyle, R., \& Fowler, W. A. 1965, in Quasi-Stellar Sources and Gravitational Collapse, ed. I. Robinson, A. Schild, \& E. L. Shucking (Chicago: Univ. Chicago Press), 17

in't Zand, J. J. M., Cornelisse, R., \& Cumming, A. 2004, A\&A, 426, 257

in 't Zand, J. J. M., Cumming, A., van der Sluys, M. V., et al. 2005, A\&A, 441, 675

Jahoda, K., Swank, J. H., Giles, A. B., et al. 1996, Proc. SPIE, 2808, 59

Kaplan, D., \& Chakrabarty, D. 2008, Astr. Tel., 1630

Kuulkers, E. 2004, Nucl. Phys. B, 132, 466

Kuulkers, E., Homan, J., van der Klis, M., et al. 2002, A\&A, 382, 947

Kuulkers, E., den Hartog, P. R., in’t Zand, J. J. M., et al. 2003, A\&A, 399, 663

Lewin, W. H. G. L., van Paradijs, J., \& Taam, R. 1993, Space Sci. Rev., 62, 223

Linares, M., et al. 2008a, Astr. Tel., 1618

Linares, M., et al. 2008b, MNRAS, in press [arXiv: 0808. 3950]

Markwardt, C. B., Pereira, D., \& Swank, J. H. 2008a, Astr. Tel., 1569

Markwardt, C. B., Cummings, J., \& Krimm, H. 2008b, Astr. Tel., 1616

Molkov, S., Revnivtsev, M., Lutovinov, A., \& Sunyaev, R. A. 2005, A\&A, 434, 1069

Peng, F., Brown, E. F., \& Truran, J. W. 2007, ApJ, 654, 1022

Rothschild, R. E., Blanco, P. R., Gruber, D. E., et al. 1998, ApJ, 496, 538

Sakamoto, T., et al. 2008, GCN circ., 8034

Starling, R., \& Evans, P. 2008, Astr. Tel., 1621

Strohmayer, T. E., \& Bildsten, L. 2006, in Compact stellar X-ray sources, ed. W.

H. G. Lewin, \& M. van der Klis (Cambridge: Cambridge University Press)

Strohmayer, T. E., Markwardt, C. B., \& Swank, J. H. 2008, Astr. Tel., 1635

Swank, J., \& Markwardt, C. 2001, in New Century of X-ray Astronomy, ed. H. Inoue, \& H. Kunieda (San Francisco: ASP), ASP Conf. Ser., 251, 94

Wallace, R. K., \& Woosley, S. E. 1981, ApJS, 45, 1389

Woosley, S. E., Heger, A., Cumming, A., et al. 2004, ApJS, 151, 75 